\title{
LETTER \\ A Simple Expression of BER Performance in DPSK/OFDM Systems with Post-Detection Diversity Reception*
}

\author{
Fumihito SASAMORI $^{\dagger \mathrm{a})}$, Shiro HANDA ${ }^{\dagger}$, and Shinjiro OSHITA $^{\dagger}$, Members
}

SUMMARY In this letter, we propose a simple but accurate calculation method, that is, an approximate closed-form equation of average bit error rate in DPSK/OFDM systems with post-detection diversity reception over both time- and frequency-selective Rayleigh fading channels. The validity of the proposed method is verified by the fact that BER performances given by the derived equation coincide with those by Monte Carlo simulation. key words: DPSK/OFDM, post-detection diversity, Doppler frequency shift, delay spread, closed-form equation

\section{Introduction}

Orthogonal frequency division multiplexing (OFDM) has intensively been studied because of the advantages of high spectrum efficiency and resistance to frequency-selective fading. Differential phase shift keying (DPSK) is widely used on the benefit of robustness and easy implementation over fading channels. DPSK/OFDM systems have the merit of applying differential modulation either in the time domain (DMT) or frequency domain (DMF) depending on the condition of fading channels [1]. Meanwhile, diversity techniques are also effective against the fading. Post-detection diversity reception is attractive because a complicated cophasing process is not required [2]. Theoretical analyses for DPSK with post-detection diversity reception have been conducted with accuracy but complexity [3], [4].

In this letter, we propose a simple but accurate closed-form equation of average bit error rate (BER) in DPSK/OFDM systems with post-detection diversity reception over both time- and frequency-selective Rayleigh fading channels. A closed-form equation can be helpful in design and development of wireless systems, because it is important to have a quantitative understanding of influence upon BER without any time-consuming simulation. A simple equation can be easily applied to BER analysis in advanced systems [5].

\section{System Model}

At a transmitter, a binary data sequence is converted into OFDM symbols with DPSK applying differential encoding in the time or frequency domain (See Figs. 1 and 2 in [1] for

\footnotetext{
Manuscript received October 29, 2008.

${ }^{\dagger}$ The authors are with the Faculty of Engineering, Shinshu University, Nagano-shi, 380-8553 Japan.

*This work was supported by MEXT, Grants-in-Aid for Young Scientists (B) 18760269.

a)E-mail: fsasa@shinshu-u.ac.jp

DOI: 10.1587/transcom.E92.B.1897
}

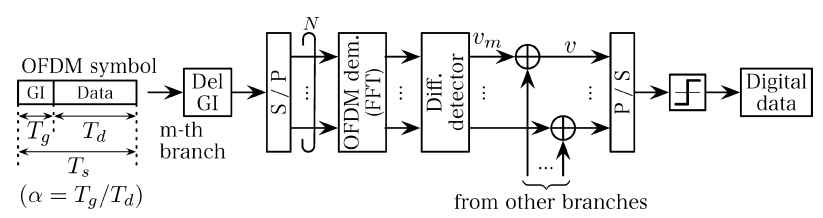

Fig. 1 Block diagram at receiver in equivalent low-pass system.

a detailed transmitting process). OFDM symbols are subjected to both time- and frequency-selective Rayleigh fading, and are added with white Gaussian noise (AWGN). The fading channel is simulated using the Jakes' model [6]. It is assumed that inter-symbol interference (ISI) caused by delay spread can be completely avoided with guard interval (GI), which is longer than the maximum multipath delay. Figure 1 shows a block diagram at a receiver, where $N$ is IFFT/FFT size, $T_{s}$ is OFDM symbol duration including GI duration $T_{g}$ and data symbol duration $T_{d}$, and $\alpha$ is a GI factor ( $\left.=T_{g} / T_{d}\right)$. A desired binary data sequence is demodulated by only deciding the signal $v$ which is produced by combining the output $v_{m}$ from each time- or frequency-domain differential detector. $\mathrm{M}$ branches combining with mutual independence is assumed in this letter. In this point, the space diversity in Fig. 1 can be replaced by the time or frequency diversity at only one receiver [5], if the fading correlation among DPSK symbols can be neglected.

\section{Statistical Analysis of BER Performance}

We have derived a closed-form equation of BER without any diversity technique [1]. In this section, we will extend the previous work to obtain the BER expression including the post-detection diversity reception.

\subsection{Closed-Form Equation of BER}

A signal $v_{m}(m=1,2, \cdots, M)$ in Fig. 1 is an output from differential detector in an arbitrary subcarrier at an arbitrary branch \#m. In the previous work [1], the signal $v_{m}$ has been expressed as

$$
\begin{aligned}
v_{m} & =\frac{1}{4}\left\{\left|z_{r\lrcorner m}+z_{d\lrcorner m}\right|^{2}-\left|z_{r\lrcorner m}-z_{d\lrcorner m}\right|^{2}\right\} \\
& \triangleq \frac{1}{4}\left(r_{1 \_m}^{2}-r_{2\lrcorner m}^{2}\right),
\end{aligned}
$$

where $z_{r\lrcorner m}$ and $z_{d \_m}$ are reference and demodulating DPSK symbols, respectively. A binary data sequence can be obtained by deciding the signal $v$ which is given by summing 
up all signals $v_{m}$ :

$$
\begin{aligned}
v & =\sum_{m=1}^{M} v_{m}=\frac{1}{4}\left(\sum_{m=1}^{M} r_{1\lrcorner m}^{2}-\sum_{m=1}^{M} r_{2\lrcorner m}^{2}\right) \\
& \triangleq \frac{1}{4}\left(r_{1}-r_{2}\right) .
\end{aligned}
$$

The BER $P_{b}$ in the binary DPSK (DBPSK) can be expressed as

$$
\begin{aligned}
P_{b} & =\operatorname{Prob}(v<0)=\operatorname{Prob}\left(r_{1}<r_{2}\right) \\
& =\int_{0}^{\infty} p\left(r_{1}\right) \int_{r_{1}}^{\infty} p\left(r_{2}\right) d r_{2} d r_{1},
\end{aligned}
$$

where $p(\cdot)$ is a probability distribution function (PDF). In order to calculate (3), it is necessary to clarify the PDFs of $r_{1}$ and $r_{2}$. Since both $r_{1\lrcorner m}$ and $r_{2\lrcorner m}$ in (2) have Rayleigh distribution, both $r_{1}$ and $r_{2}$ have Gamma distribution [6].

Now we will derive the mean values and variances of $r_{1}$ and $r_{2}$. From (2), the mean values $E\left[r_{1}\right]$ and $E\left[r_{2}\right]$ can be expressed as

$$
\begin{aligned}
& E\left[r_{1}\right]=\sum_{m=1}^{M} E\left[r_{1\lrcorner m}^{2}\right]=M\left\{2 \sigma_{s}^{2}(1+\rho)+2 \sigma_{n}^{2}\right\} \\
& E\left[r_{2}\right]=\sum_{m=1}^{M} E\left[r_{2\lrcorner m}^{2}\right]=M\left\{2 \sigma_{s}^{2}(1-\rho)+2 \sigma_{n}^{2}\right\} .
\end{aligned}
$$

In the above equation, $\sigma_{s}^{2}$ and $\sigma_{n}^{2}$ mean the variances of the signal over Rayleigh fading channels and the AWGN, respectively. $\rho$ means the fading correlation between adjacent DPSK symbols in the time domain (DMT) or the frequency domain (DMF). Assuming the Jakes' model, $\rho$ is given by

$$
\rho= \begin{cases}J_{0}\left(2 \pi f_{D} T_{s}\right) & \text { for DMT } \\ \frac{1}{\sqrt{1+\left\{2 \pi(1+\alpha) \frac{\sigma_{\tau}}{T_{s}}\right\}^{2}}} & \text { for DMF, }\end{cases}
$$

where $f_{D}$ and $\sigma_{\tau}$ are the maximum Doppler frequency and the rms delay spread, respectively. We should note that $f_{D}$ and $\sigma_{\tau}$ have no influence upon DMF and DMT, respectively. We now define

$$
\begin{aligned}
& \sigma_{1}^{2} \triangleq 2 \sigma_{s}^{2}(1+\rho)+2 \sigma_{n}^{2} \\
& \sigma_{2}^{2} \triangleq 2 \sigma_{s}^{2}(1-\rho)+2 \sigma_{n}^{2} .
\end{aligned}
$$

Based on the characteristic of Gamma distribution, the mean values and variances of $r_{1}$ and $r_{2}$ are given by

$$
\begin{aligned}
& E\left[r_{i}\right]=M \sigma_{i}^{2} \\
& E\left[\left(r_{i}-E\left[r_{i}\right]\right)^{2}\right]=M \sigma_{i}^{4} \quad(i=1,2) .
\end{aligned}
$$

By using Gamma function $\Gamma(\cdot)$, the PDFs of $r_{1}$ and $r_{2}$ can be written as

$$
p\left(r_{i}\right)=\frac{r_{i}^{M-1}}{\Gamma(M)\left(\sigma_{i}^{2}\right)^{M}} e^{-\frac{r_{i}}{\sigma_{i}^{2}}} \quad(i=1,2) .
$$

By substituting (8) into (3), the BER can be solved as follows (See Appendix for the derivation method):

$$
\begin{aligned}
P_{b}= & \frac{1}{(M-1) !} \\
& \times \sum_{m=0}^{M-1} \frac{(2 M-2-m) !}{(M-1-m) !} \frac{\left(\frac{\sigma_{1}^{2}}{\sigma_{2}^{2}}\right)^{M-1-m}}{\left(\frac{\sigma_{1}^{2}}{\sigma_{2}^{2}}+1\right)^{2 M-1-m} .}
\end{aligned}
$$

\subsection{Approximation}

From (6), $\sigma_{1}^{2} / \sigma_{2}^{2}$ in (9) can be rewritten as

$$
\frac{\sigma_{1}^{2}}{\sigma_{2}^{2}}=\frac{(1+\rho) \Gamma_{E N}^{\prime}+1}{(1-\rho) \Gamma_{E N}^{\prime}+1} \quad\left(\Gamma_{E N}^{\prime} \triangleq \sigma_{s}^{2} / \sigma_{n}^{2}\right),
$$

where $\sigma_{s}^{2} / \sigma_{n}^{2}$ and $\Gamma_{E N}^{\prime}$ mean the carrier-to-noise power ratio (CNR) and the ratio of energy per bit to the spectral noise density $\left(E_{b} / N_{0}\right)$ on a received signal per branch in the case of the DBPSK, respectively. It should be noted that $\Gamma_{E N}^{\prime}$ includes both inter-carrier interference and intra-symbol interference, which is mentioned later. $\Gamma_{E N}^{\prime}$ and $\rho$ generally satisfy $\Gamma_{E N}^{\prime} \gg 1$ and $\rho \simeq 1$, then those facts yield $\sigma_{1}^{2} / \sigma_{2}^{2} \gg 1$. In (9), we consequently get the following approximation:

$$
\frac{\left(\frac{\sigma_{1}^{2}}{\sigma_{2}^{2}}\right)^{M-1-m}}{\left(\frac{\sigma_{1}^{2}}{\sigma_{2}^{2}}+1\right)^{2 M-1-m}} \simeq \frac{1}{\left(\frac{\sigma_{1}^{2}}{\sigma_{2}^{2}}+1\right)^{M}},
$$

which becomes irrelevant to $m$. Moreover, by using the following formula:

$$
\sum_{x=1}^{n} x(x+1) \cdots(x+k)=\frac{1}{k+2} \frac{(n+k+1) !}{(n-1) !},
$$

the summation term in (9) can be rewritten as

$$
\sum_{m=0}^{M-1} \frac{(2 M-2-m) !}{(M-1-m) !}=\frac{(2 M-1) !}{M !} .
$$

By substituting (11) and (13) into (9), we finally obtain a simple closed-form equation of BER:

$$
P_{b}=\frac{(2 M-1) !}{M !(M-1) !} \frac{1}{\left(\frac{\sigma_{1}^{2}}{\sigma_{2}^{2}}+1\right)^{M}} .
$$

We should note that the expressions (9) and (14) in the case of non-diversity $(M=1)$ coincide with that of the previous work [1].

Figure 2 shows the BER performance without GI over quasi-static fading channels, that is, without any inter-carrier interference and any intra-symbol interference $\left(\Gamma_{E N}^{\prime}=\Gamma_{E N}\right.$, $\rho=1)$. From (10) and (14), the approximation result can be obtained as follows:

$$
P_{b}=\frac{(2 M-1) !}{M !(M-1) !} \frac{1}{2^{M}\left(\Gamma_{E N}+1\right)^{M}},
$$

which indicates the same performance in the case of single 
carrier systems. Besides approximation and simulation results, the performances of pre-detection MRC in the case of $M=2,4$ are simultaneously shown:

$$
P_{b_{-} p r e}=\frac{1}{2\left(\Gamma_{E N}+1\right)^{M}} .
$$

From (15) and (16), it can be theoretically verified that the BER performance of two-branch $(M=2)$ post-detection MRC is about $0.9 \mathrm{~dB}$ inferior to that of pre-detection MRC [2].

\subsection{Interference Analysis}

We have investigated two kinds of interferences [1]: intercarrier interference $I_{a}$ caused by Doppler frequency and intra-symbol interference (interference to the quadrature channel) $I_{d}$ caused by Doppler frequency or delay spread in the case of DQPSK only. The $I_{a}$ and $I_{d}$ have been derived as follows:

$$
\begin{aligned}
I_{a} & =\frac{\left(\pi f_{D} T_{s}\right)^{2}}{6(1+\alpha)^{2}}, \\
I_{d} & = \begin{cases}0 & \text { for DBPSK } \\
\frac{\left(\pi f_{D} T_{s}\right)^{2}}{2} & \text { for DQPSK(DMT) } \\
\left\{\pi(1+\alpha) \frac{\sigma_{\tau}}{T_{s}}\right\}^{2} & \text { for DQPSK(DMF). }\end{cases}
\end{aligned}
$$

When combining the signals of $\mathrm{M}$ branches which are mutually independent on Doppler fluctuation, the signal of one branch also suffers from inter-carrier interferences of the other $(M-1)$ branches. Since the fluctuated angle $\theta$ by Doppler shows an uniform distribution on $[0,2 \pi]$, the interbranch interference $I_{b}$ can be calculated as

$$
\begin{aligned}
I_{b} & =\frac{1}{2 \pi} \int_{0}^{2 \pi}\left(\sqrt{I_{a}} \cos \theta\right)^{2} d \theta \times(M-1) \\
& =\frac{(M-1)\left(\pi f_{D} T_{S}\right)^{2}}{12(1+\alpha)^{2}} .
\end{aligned}
$$

By using Gaussian approximation [7] and taking the relation between $E_{b} / N_{0}$ and CNR into consideration, $\Gamma_{E N}^{\prime}$ in (10) can be obtained as follows:

$$
\Gamma_{E N}^{\prime}= \begin{cases}\frac{1}{\frac{1+\alpha}{\Gamma_{E N}+I_{a}+I_{b}}} & \text { for DBPSK } \\ \frac{1}{\frac{1+\alpha}{\Gamma_{E N}}+2\left(I_{a}+I_{d}+I_{b}\right)} & \text { for DQPSK, }\end{cases}
$$

where $(1+\alpha)$ means the loss of energy when removing GI. Finally, substituting (5), (10), (17)-(20) into (14) leads the approximate closed-form equation of the BER. This method can simply calculate the average BER from the following parameters: $\Gamma_{E N}\left(E_{b} / N_{0}\right), f_{D} T_{s}, \sigma_{\tau} / T_{s}, M$ and $\alpha$.

The DMF and DMT are sensitive to delay spread and Doppler frequency, respectively, then we evaluate the influence of them in Fig. 3 with (14) and simulation. As for a delay profile model, an exponential decaying model is assumed. From both approximation and simulation results, the performances of DMF and DMT begin degrading at

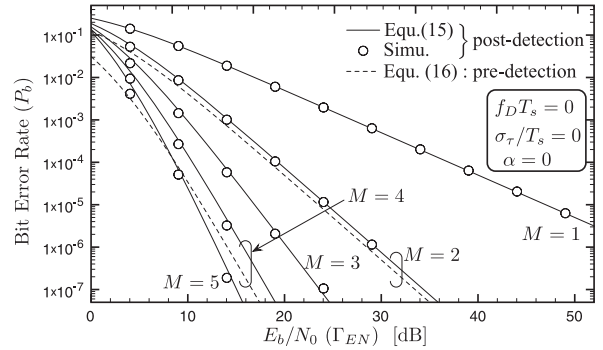

Fig. 2 BER performance over quasi-static fading channels.

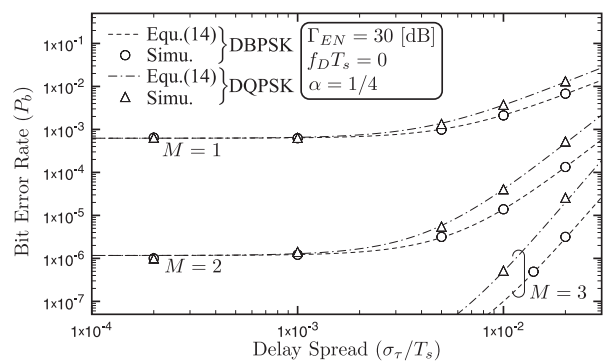

(a) BER versus $\sigma_{\tau} / T_{s}$ in DMF

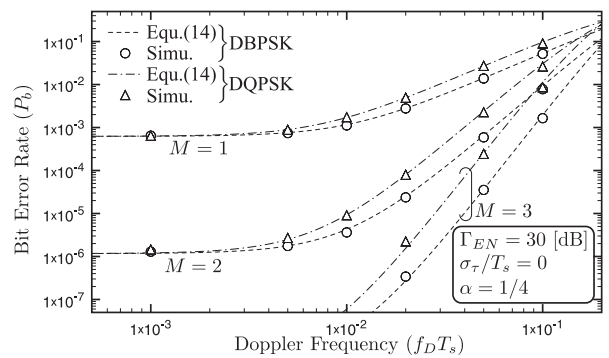

(b) BER versus $f_{D} T_{s}$ in DMT

Fig. 3 BER sensitivity to $\sigma_{\tau} / T_{s}$ and $f_{D} T_{s}$.

$\sigma_{\tau} / T_{s}=2 \times 10^{-3}$ and $f_{D} T_{s}=3 \times 10^{-3}$, respectively. It is found from Figs. 2 and 3 that the analysis in Section 3 agrees with Monte Carlo simulation. In order to make the validity of analysis steadier, numerical evaluations over typical channel models should be also conducted.

\section{Numerical Evaluations over Typical Channel Mod- els}

The BER performances assuming IMT-2000 vehicular channel A and ETSI/BRAN channel A are shown in Fig. 4 (See [1] for detailed simulation parameters). Irrespective of frequency selectivity, the DMT has a disadvantage over severe time-selective fading like IMT-2000 vehicular channel models. In other words, the DMF is attractive for mobile communications. But the DMT is attractive for fixed communications like ETSI/BRAN Channel A. The proposed simple equation can precisely and quantitatively express the above characteristics.

\section{Conclusion}

We have proposed a simple closed-form equation of the av- 


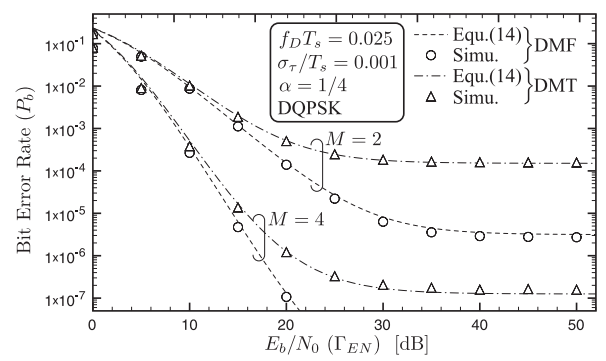

(a) Over IMT-2000 vehicular channel A

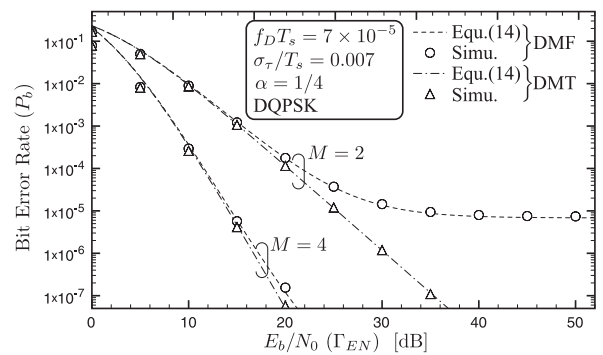

(b) Over ETSI/BRAN channel A

Fig. 4 BER performances over typical channel models.

erage BER in DPSK/OFDM systems with post-detection diversity reception over both time- and frequency-selective Rayleigh fading channels. It has been confirmed by the numerical evaluation that the proposed equation can precisely express the characteristics of BER performance.

\section{References}

[1] F. Sasamori, S. Handa, and S. Oshita, "A simple method of BER calculation in DPSK/OFDM systems over fading channels," IEICE Trans. Fundamentals, vol.E88-A, no.1, pp.366-373, Jan. 2005.

[2] F. Adachi and K. Ohno, "BER performance of QDPSK with postdetection diversity reception in mobile radio channels," IEEE Trans. Veh. Technol., vol.40, no.1, pp.237-249, Feb. 1991.

[3] Y. Ma and Q.T. Zhang, "Accurate evaluation for MDPSK with noncoherent diversity," IEEE Trans. Commun., vol.50, no.7, pp.11891200, July 2002.

[4] Q.T. Zhang, "Exact analysis of postdetection combining for DPSK and NFSK systems over arbitrarily correlated Nakagami channels," IEEE Trans. Commun., vol.46, no.11, pp.1459-1467, Nov. 1998.

[5] F. Sasamori, Y. Ishikawa, S. Handa, and S. Oshite, "A simple expression of BER performance in COFDM systems over fading chan- nels," IEICE Trans. Fundamentals, vol.E92-A, no.1, pp.332-336, Jan. 2009.

[6] W.C. Jakes, Microwave Mobile Communications, IEEE Press, New York, 1974.

[7] T.S. Rappaport, Wireless Communications: Principles and Practice, Prentice Hall, 1996.

\section{Appendix: Derivation Method of (9)}

In the process of integration in (3), the following formula is utilized:

$$
\int r^{n} e^{-\frac{r}{a}} d r=-a e^{-\frac{r}{a}} \sum_{m=0}^{n} \frac{a^{m} r^{n-m} n !}{(n-m) !} .
$$

First of all, we conduct the following integration:

$$
\begin{aligned}
& \int_{r_{1}}^{\infty} p\left(r_{2}\right) d r_{2} \\
& =\frac{1}{\left(\sigma_{2}^{2}\right)^{M-1}} e^{-\frac{r_{1}}{\sigma_{2}^{2}}} \sum_{m=0}^{M-1} \frac{\left(\sigma_{2}^{2}\right)^{m} r_{1}^{M-1-m}}{(M-1-m) !}
\end{aligned}
$$

where Gamma function can be rewritten as $\Gamma(M)=(M-1)$ ! if $M$ is a natural number. By substituting (A-2) into (3) and rearranging it, we obtain

$$
\begin{aligned}
P_{b}= & \frac{1}{\Gamma(M)\left(\sigma_{1}^{2}\right)^{M}\left(\sigma_{2}^{2}\right)^{M-1}} \sum_{m=0}^{M-1}\left\{\frac{\left(\sigma_{2}^{2}\right)^{m}}{(M-1-m) !}\right. \\
& \left.\times \int_{0}^{\infty} r_{1}^{2 M-2-m} e^{-\left(\frac{1}{\sigma_{1}^{2}}+\frac{1}{\sigma_{2}^{2}}\right) r_{1}} d r_{1}\right\} .
\end{aligned}
$$

By defining $n \triangleq 2 M-2-m$ and $a \triangleq\left(1 / \sigma_{1}^{2}+1 / \sigma_{2}^{2}\right)^{-1}$, the integral part with respect to $r_{1}$ in (A.3) can be solved as follows:

$$
\int_{0}^{\infty} r_{1}^{n} e^{-\frac{r_{1}}{a}} d r_{1}=a \sum_{m^{\prime}=0}^{n} \frac{a^{m^{\prime}} 0^{n-m^{\prime}} n !}{\left(n-m^{\prime}\right) !}=a^{n+1} n !,
$$

because $0^{n-m^{\prime}}=1$ only if $m^{\prime}=n$. By rearranging (A. 3) with (A.4), we obtain (9). 